\title{
Measurements with doubly-charmed hadrons in LHCb
}

\author{
Daniel Vieira ${ }^{\dagger 1, *}$ \\ ${ }^{1}$ University of Chinese Academy of Sciences (UCAS), Beijing, China
}

\begin{abstract}
The LHCb experiment has been collecting large amounts of charm data, making it possible to perform precise measurements and search for rare particles. In 2017, the LHCb collaboration reported the first observation of the doubly charmed baryon, the $\Xi_{c c}^{++}$. In 2018, the first measurement of the $\Xi_{c c}^{++}$lifetime, using the same decay and dataset, was published. This paper summarizes the content of both analyses, as reported in the 9th International Workshop on Charm Physics, in Novosibirsk, Russia.
\end{abstract}

\section{Introduction}

The quark model predicts the existence of two SU(4) 20-plets of baryons containing $u, d, s$ and $c$ quarks, one with $J^{P}=1 / 2^{+}$and the other with $J^{P}=3 / 2^{+}$. While baryons from the first multiplet decay weakly, baryons from the second one decay via strong interactions with very short lifetime. Each of the 20-plets contain a SU(3) triplet of doubly charmed baryons, the $\Xi_{c c}^{++}(c c u)$, the $\Xi_{c c}^{+}(c c d)$ and the $\Omega_{c c}^{+}(c c s)$. The doubly charmed baryons have sparked great interest both from the experimental and theoretical point of view, as a promising environment to test calculations from the Standard Model (SM) of particle physics. Many predictions are available in literature regarding the properties of doubly charmed baryons, such as for their mass [1-13] and lifetime [2, 9, 14-21]. In particular, the mass of the $\Xi_{c c}^{+}$and $\Xi_{c c}^{++}$are expected to be around the same value, and to fall between 3.5 and $3.7 \mathrm{GeV} / c^{2}$. On the other hand, the lifetime of the $\Xi_{c c}^{++}$is predicted to be between $0.185 \mathrm{ps}$ and $1.050 \mathrm{ps}$, and to be around three times larger than the $\Xi_{c c}^{+}$and $\Omega_{c c}^{+}$.

The SELEX collaboration has reported the observation of the $\Xi_{c c}^{+}$in two different channels, $\Xi_{c c}^{+} \rightarrow \Lambda_{c}^{+} K^{-} \pi^{+}$[22] and $\Xi_{c c}^{+} \rightarrow p D^{+} K^{-}$[23]. Searches from FOCUS [24], BaBar [25], Belle [26] and LHCb [27] were not able to confirm the findings, however. More searches are needed to resolve this experimental puzzle. Once discovered, measurements of the properties of such particles are needed in order to establish the nature of their decay, while also providing an important test to theoretical predictions. An alternative approach, motivated by recent theoretical calculations [28], is to search for $\Xi_{c c}^{++}$decays to $\Lambda_{c}^{+} K^{-} \pi^{+} \pi^{+}$and $\Xi_{c}^{+} \pi^{+}$, due to their significant branching fractions.

This document reports two recent results from the $\mathrm{LHCb}$ collaboration. In the first one, a peak is observed in the $\Lambda_{c}^{+} K^{-} \pi^{+} \pi^{+}$invariant mass spectrum, where the $\Lambda_{c}^{+}$decays to $p K^{-} \pi^{+}$, compatible with a weakly decaying $\Xi_{c c}^{++}$[29]. The second result is the lifetime measurement of the $\Xi_{c c}^{++}[30]$.

\footnotetext{
$\dagger$ On behalf of LHCb collaboration

*e-mail: devieira@cern.ch
} 


\section{The LHCb experiment}

The LHCb detector $[31,32]$ is a single-arm forward spectrometer designed for measuring decays of particles containing $c$ and $b$ quarks. For hadron spectroscopy studies, such as the search for the $\Xi_{c c}^{++}$, some detector elements are particularly important: a silicon-strip based vertex detector [33], located close to the pp collision region, provides excellent vertex reconstruction, essential to separate the collision position from displaced vertices, typical in $c$ and $b$ decays; a high precision tracking system [34], which provides great momentum resolution for charged particles; and two-ring-imaging Cherenkov detectors [35], responsible for discriminating different species of charged hadrons. The magnet polarity is periodically inverted, allowing for systematic studies and canceling eventual detection asymmetries. A trigger system composed by two stages performs the event selection during the data acquisition [36-38]. The first stage is done on a hardware level and is based on the energy deposits measured in calorimeters and muon stations. The second stage is performed on software level and depends on the full-event reconstruction with near-real-time alignment and calibration of the detector.

Both analyses reported in this document use the 2016 dataset from the LHCb experiment. It consists of a sample of proton-proton collisions with centre-of-mass energy of $13 \mathrm{TeV}$, corresponding to an integrated luminosity of approximately $1.7 \mathrm{fb}^{-1}$.

\section{Observation of $\Xi_{c c}^{++} \rightarrow \Lambda_{c}^{+} K^{-} \pi^{+} \pi^{+}$}

The $\Xi_{c c}^{++} \rightarrow \Lambda_{c}^{+} K^{-} \pi^{+} \pi^{+}$selection is done in three stages:

- Trigger - selection is performed as the decay is reconstructed;

- Preselection - a set of loose selection criteria is applied focusing in reducing combinatorial background;

- Multivariate Analysis (MVA) - a multivariate selection based on the multilayer perceptron algorithm (MLP) [39] is applied to improve the signal-to-background ratio.

The reconstruction is initially performed by combining a proton, a $K^{-}$and a $\pi^{+}$to form the $\Lambda_{c}^{+}$candidate. Afterwards, the $\Lambda_{c}^{+}$candidate is combined with an extra $K^{-}$, a $\pi^{+}$and a $\pi^{+}$, forming the $\Xi_{c c}^{++}$candidate. A number of selection criteria is applied in each of these steps and after the full reconstruction, mostly based on the decay topology, i.e., that the trajectory of combined particles form a vertex with good fit quality in each step, its kinematic variables and the consistency with the corresponding particle identification hypothesis. The $\Lambda_{c}^{+}$candidate reconstructed mass $m_{\text {cand }}\left(\Lambda_{c}^{+}\right)$is required to be within the limits $2270<m_{\text {cand }}\left(\Lambda_{c}\right)<2306 \mathrm{MeV} / c^{2}$, while the $\Xi_{c c}^{++}$reconstructed mass $m_{\text {cand }}\left(\Xi_{c c}^{++}\right)$, defined as $m_{\text {cand }}\left(\Xi_{c c}^{++}\right) \equiv m\left(\Lambda_{c}^{+} K^{-} \pi^{+} \pi^{+}\right)-m_{\text {cand }}\left(\Lambda_{c}^{+}\right)+m_{\mathrm{PDG}}\left(\Lambda_{c}^{+}\right)$, where $m\left(\Lambda_{c}^{+} K^{-} \pi^{+} \pi^{+}\right)$is the combined $\Lambda_{c}^{+} K^{-} \pi^{+} \pi^{+}$mass and $m_{\mathrm{PDG}}\left(\Lambda_{c}^{+}\right) s$ is the $\Lambda_{c}$ mass value given in PDG [40], is required to be within $3300<m_{\text {cand }}\left(\Xi_{c c}^{++}\right)<3800 \mathrm{MeV} / c^{2}$.

The MLP selection is trained with centrally produced LHCb Monte Carlo simulation, using the GenXicc package $[41,42]$ for the $\Xi_{c c}^{++}$baryon production, together with Pythia [43, 44] (with a specific LHCb configuration), EvtGen [45], PHOTOS [46], and Geant4 [47, 48], in order to represent our signal events. The background is represented by a wrong-sign (WS) sample of $\Lambda_{c}^{+} K^{-} \pi^{+} \pi^{-}$in the training. Both are required to have passed all trigger and preselection criteria before the training. The MLP takes into account, again, a series of kinematical and topological properties of the decays, such as the vertex fit quality and the quality of kinematical refits done under the hypothesis that the $\Xi_{c c}^{++}$is originated at the collision point [49]. The chosen cut value of MLP output is the one which maximizes the Punzi figure of merit [50]. 
After all selection criteria are applied, a visible peaking structure in the $m_{\text {cand }}\left(\Xi_{c c}^{++}\right)$distribution can be seen around $3620 \mathrm{MeV} / c^{2}$ as seen in Figure 1. This structure is not present in the WS sample. The mass peak over the signal region is fitted with a sum of a Gaussian function and a Double-sided Crystal ball function [51]. The background is described by a second order Chebyshev polynomial. The fit provides an effective mean mass value of $(3621.40 \pm 0.72$ (stat.) \pm 0.31 (syst.) $) \mathrm{MeV} / c^{2}$, with a yield of $313 \pm 33$ signal events and a mass resolution of $(6.6 \pm 1.4) \mathrm{MeV} / c^{2}$. consistent with the experimental resolution seen in MC. The obtained mass value is within the range of theoretical predictions, but significantly different from the SELEX $\Xi_{c c}^{+}$measurement.

Many systematic studies and crosschecks are performed. The main systematic contributions to the mass measurement are due to the uncertainty on the PDG $\Lambda_{c}^{+}$mass, the momentum scale calibration and the correction of the selection criteria bias. The observation is crosschecked, among other tests, with a fully independent dataset, taken by LHCb in 2012, with $8 \mathrm{TeV}$ centre-of-mass energy, corresponding to an integrated luminosity of $2.0 \mathrm{fb}^{-1}$. Around $113 \pm 13$ events are seen with consistent mass and resolution values.

Given the solid observation of a structure consistent with the $\Xi_{c c}^{++} \rightarrow \Lambda_{c}^{+} K^{-} \pi^{+} \pi^{+}$decay, it is possible to make further studies, in order to understand the nature of this particle. In particular, measuring its lifetime is crucial to understand if the particle decays via the weak or the strong interaction. In the observation analysis, a cross check is performed where the data is required to have a decay time five times larger than the decay time resolution in LHCb. Even when making this requirement, the mass peak is still significant, which indicates the decay has a weak nature, but only a lifetime measurement is able to precisely establish it.

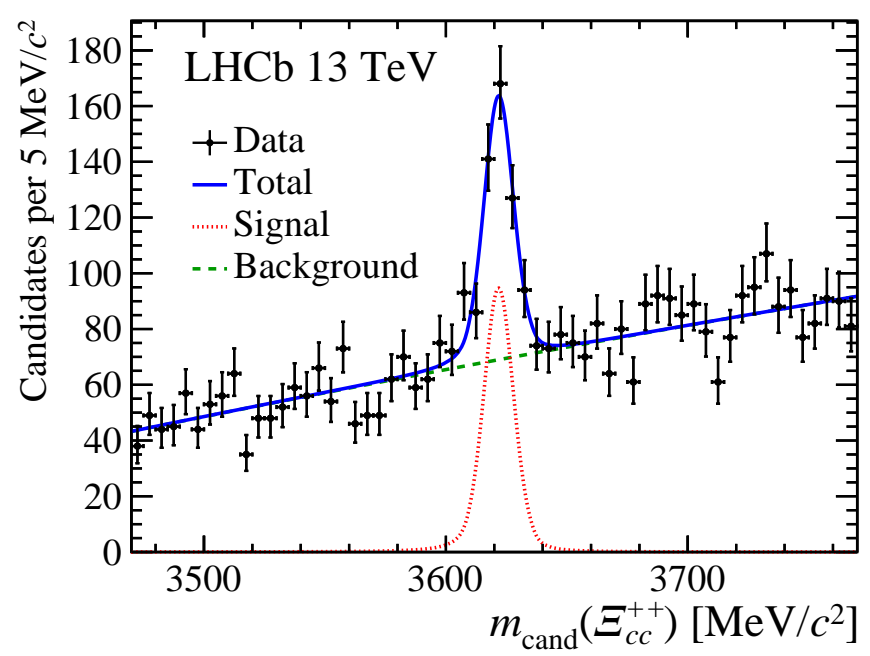

Figure 1. Invariant mass distribution of $\Xi_{c c}^{++} \rightarrow \Lambda_{c}^{+} K^{-} \pi^{+} \pi^{+}$candidates with the corresponding fit function.

\section{Measurement of the $\Xi_{c c}^{++}$lifetime}

The lifetime measurement of the $\Xi_{c c}^{++}$is performed on the same dataset and decay used for the observation. Only a couple of additional requirements are added, in order to have better control over the efficiency. The events are required to be selected on the hardware trigger 
level either by a large energy deposit on the hadronic calorimeter from one of the final state particles or by any energy deposit from other particles. Additionally, the candidates are required to have a decay time larger than $0.1 \mathrm{ps,} \mathrm{in} \mathrm{order} \mathrm{to} \mathrm{avoid} \mathrm{resolution} \mathrm{effects,} \mathrm{and}$ smaller than $2.0 \mathrm{ps}$, after which the signal sample contains almost no entries.

The $\Xi_{c c}^{++}$decay time is measured relative to the $\Lambda_{b} \rightarrow \Lambda_{c}^{+} \pi^{-} \pi^{+} \pi^{-}$. Since both decays present very similar topology, systematic effects due to efficiency and reconstruction are mostly cancelled when taking the ratio between their decay time distribution. Both are required to go through the same selection criteria, with the exception of the corresponding mass windows and PID cuts. The mass distribution is fitted with the same procedure as the one done in section 3, providing a yield of $304 \pm 35$ events for $\Xi_{c c}^{++}$and $3397 \pm 119$ events for $\Lambda_{b}$. Weights are extracted from the mass fit, using the sPlot technique [52], in order to subtract the background from the decay time distribution. The mass fits can be seen in Fig. 2, while their corresponding background subtracted decay time distribution is shown in Fig. 3.
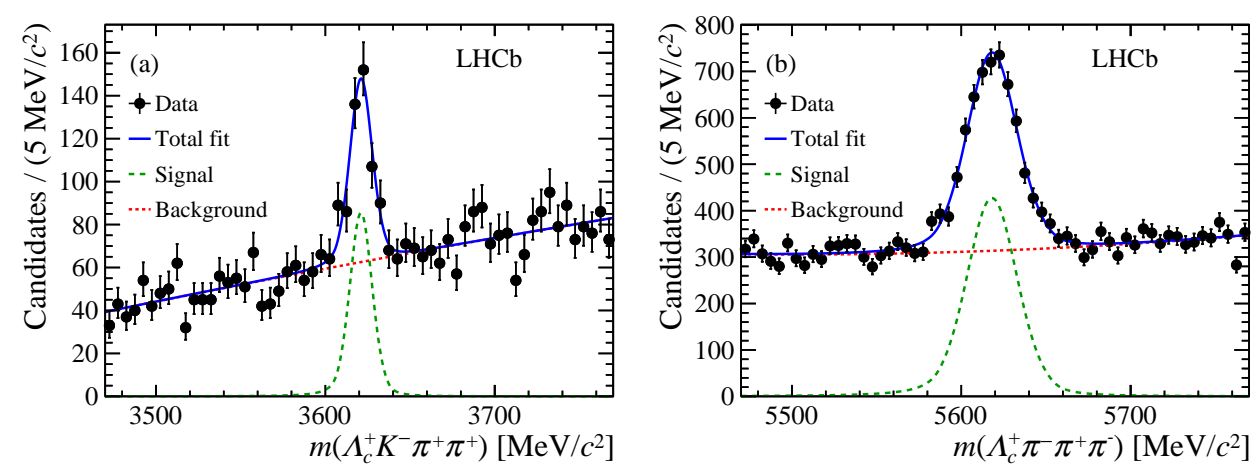

Figure 2. Invariant mass distribution of $\Xi_{c c}^{++} \rightarrow \Lambda_{c}^{+} K^{-} \pi^{+} \pi^{+}$candidates with the corresponding fit function for the lifetime measurement.

Centrally produced LHCb Monte Carlo samples, with the same tools as described in 3, are used to study the efficiencies as a function of decay time. The acceptance is determined by the ratio between reconstructed and generated decay time distributions of MC samples. The simulated samples are weighted to better reproduce the data transverse momentum distribution. The corresponding acceptances are shown in Fig. 4.

A weighted unbinned maximum likelihood fit is performed to determine the $\Xi_{c c}^{++}$lifetime. The Probability Density Function (PDF) used to describe the decay time distribution is defined as:

$$
f_{\Xi_{c c}^{++}}(t)=H_{\Lambda_{b}}(t) \times \frac{\epsilon_{\Xi_{c c}^{++}}(t)}{\epsilon_{\Lambda_{b}}(t)} \times \exp \left(\frac{t}{\tau_{\Lambda_{b}}}-\frac{t}{\tau_{\Xi_{c c}^{++}}}\right),
$$

Where $H_{\Lambda_{b}}(t)$ is the background subtracted $\Lambda_{b}$ decay time data distribution, $\epsilon_{\Xi_{c c}^{++}}\left(\Lambda_{b}\right)(t)$ is the $\Xi_{c c}^{++}\left(\Lambda_{b}\right)$ acceptance as a function of time, $\tau_{\Lambda_{b}}=1.470 \pm 0.010 \mathrm{ps}$ is the known value of the $\Lambda_{b}$ lifetime and $\tau_{\Xi_{c c}^{++}}$is the $\Xi_{c c}^{++}$lifetime to be determined in the fit. The distribution $H_{\Lambda_{b}}(t), \epsilon_{\Xi_{c c}^{++}}(t)$ and $\epsilon_{\Lambda_{b}}(t)$ are represented by histograms in the PDF, with bin sizes chosen to minimize the uncertainty due to the limited statistics in each bin. The fit result can be seen in Fig. 5 and the obtained lifetime value is $\tau\left(\Xi_{c c}^{++}\right)=\left(0.256_{-0.022}^{+0.024}\right) \mathrm{ps}$, where uncertainties are statistical only and take into account the uncertainty due to the limited size of MC samples (0.007 ps) and of the $\Lambda_{b}$ data sample (0.006 ps).

Many sources of systematic uncertainties and crosschecks are performed. The main systematic contribution is due to resonant contributions in the decay that are not taken into 


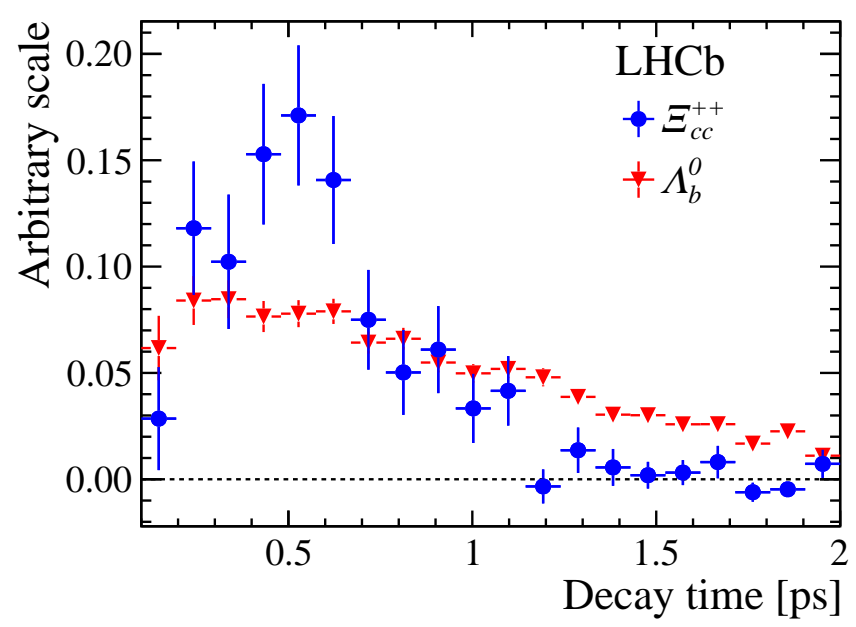

Figure 3. Background subtracted decay time distribution for the $\Xi_{c c}^{++}$(red) and $\Lambda_{b}$ (blue).

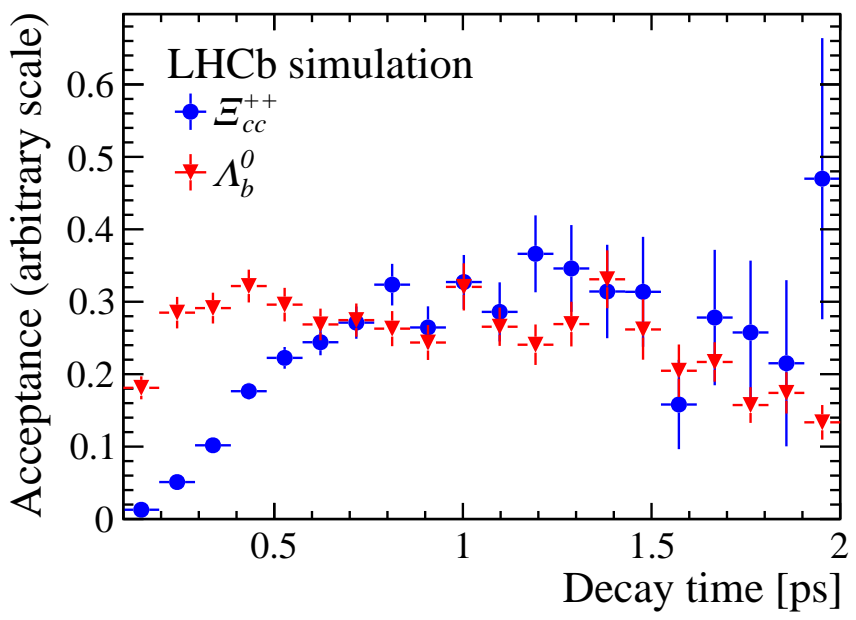

Figure 4. Decay time acceptances of the $\Xi_{c c}^{++}$(red) and $\Lambda_{b}$ (blue). Both MC samples are weighted to match the transverse momentum distribution seen in data.

account in simulation $(0.011 \mathrm{ps})$. The $\Xi_{c c}^{++} \mathrm{MC}$ sample distribution is reweighted to match the most significant resonant contribution and the difference in result is taken as systematic uncertainty. Other systematic contributions are due to the mass model used in the fit $(0.005$ $\mathrm{ps})$, the correlation between mass and decay time $(0.004 \mathrm{ps})$, the choice of bin size in the histograms $(0.001 \mathrm{ps})$, the difference in the response with respect to the multivariate selection between data and MC (0.004 ps), the impact of different trigger selections on data (0.002 ps), the $\Xi_{c c}^{++}$lifetime value used in simulation $(0.002 \mathrm{ps})$ and the uncertainty over the $\Lambda_{b}$ lifetime (0.001 ps). 


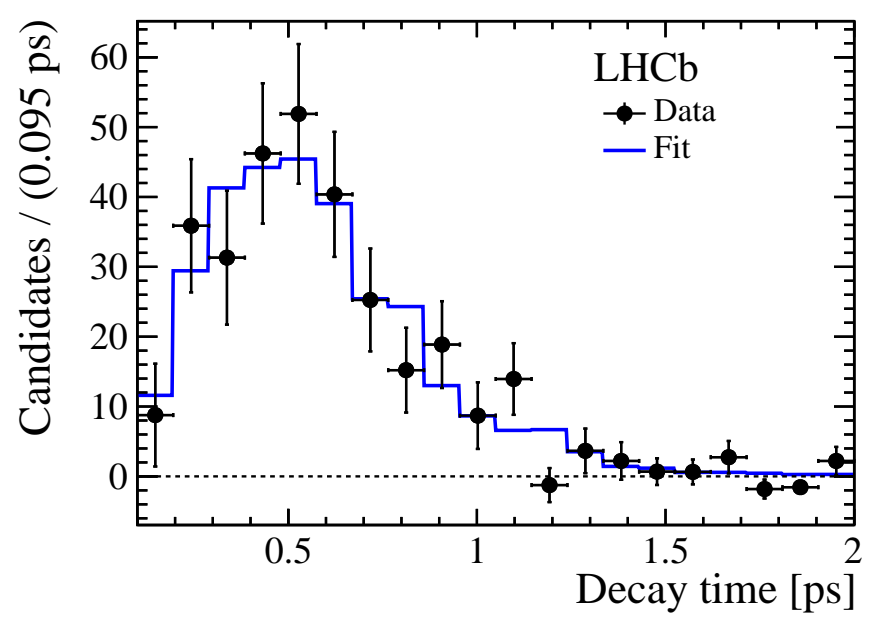

Figure 5. $\Xi_{c c}^{++}$decay distribution and fit result superimposed.

The measurement is checked to be unaffected by decay time resolution effects and by the multivariate threshold value. Subsets of the data, i.e., splitting the data by opposite LHCb magnet polarity (up and down), by charge $\left(\Xi_{c c}^{++}\right.$and $\bar{\Xi}_{c c}^{--}$) and by number of primary vertices, all provide compatible results. Finally, an alternative method is employed as a cross check, where a minimum squares binned fit is performed, instead of the unbinned maximum likelihood fit, and, once more, a consistent result is obtained. Considering all systematic results, the lifetime is measured to be $\tau\left(\Xi_{c c}^{++}\right)=\left(0.256_{-0.022}^{+0.024}\right.$ (stat.) \pm 0.014 (syst.)) ps. The measured value is within the range of theoretical predictions, and consistent with a weak decay.

\section{Conclusions}

The studies reported by $\mathrm{LHCb}$ collaboration present the observation of a structure in the $\Lambda_{c}^{+} K^{-} \pi^{+} \pi^{+}$invariant mass spectrum consistent with a weakly decaying $\Xi_{c c}^{++}$. Its mass was measured to be $\left(3621.40 \pm 0.72\right.$ (stat.) \pm 0.31 (syst.)) $\mathrm{MeV} / c^{2}$, which is within the range of theoretical predictions. On the other hand, the obtained mass value is $103 \pm 2 \mathrm{MeV} / c^{2}$ away from the SELEX $\Xi_{c c}^{+}$measurement, which would be an atypically large difference for an isospin doublet. The $\Xi_{c c}^{++}$lifetime is measured to be $\tau\left(\Xi_{c c}^{++}\right)=\left(0.256_{-0.022}^{+0.024}\right.$ (stat.) \pm 0.014 (syst.)) ps, also within the range of theoretical predictions and consistent with a weak decay.

\section{References}

[1] C. Itoh, T. Minamikawa, K. Miura, T. Watanabe, Phys. Rev. D61, 057502 (2000)

[2] K. Anikeev et al., B physics at the Tevatron: Run II and beyond, in Workshop on $B$ physics at the Tevatron: Run II and beyond, Batavia, Illinois, September 23-25, 1999 (2001), hep-ph/0201071, http://lss.fnal.gov/cgi-bin/find_paper. pl?pub-01- 197

[3] W. Roberts, M. Pervin, Int.J.Mod.Phys. A23, 2817 (2008), 0711. 2492 
[4] D.H. He, K. Qian, Y.B. Ding, X.Q. Li, P.N. Shen, Phys.Rev. D70, 094004 (2004), hep-ph/0403301

[5] Z.G. Wang, Eur.Phys.J. A45, 267 (2010), 1001.4693

[6] C.H. Chang, C.F. Qiao, J.X. Wang, X.G. Wu, Phys.Rev. D73, 094022 (2006), hep-ph/0601032

[7] A. Valcarce, H. Garcilazo, J. Vijande, Eur.Phys.J. A37, 217 (2008), 0807. 2973

[8] J.R. Zhang, M.Q. Huang, Phys.Rev. D78, 094007 (2008), 0810. 5396

[9] M. Karliner, J.L. Rosner, Phys. Rev. D90, 094007 (2014), 1408. 5877

[10] C. Alexandrou, C. Kallidonis, Phys. Rev. D96, 034511 (2017), 1704.02647

[11] K.W. Wei, B. Chen, X.H. Guo, Phys. Rev. D92, 076008 (2015), 1503.05184

[12] Z.F. Sun, M.J. Vicente Vacas, Phys. Rev. D93, 094002 (2016), 1602.04714

[13] D. Ebert, R. Faustov, V. Galkin, A. Martynenko, Phys.Rev. D66, 014008 (2002), hep-ph/0201217

[14] S. Fleck, J.M. Richard, Prog. Theor. Phys. 82, 760 (1989)

[15] B. Guberina, B. Melić, H. Štefančić, Eur.Phys.J. C9, 213 (1999), hep-ph/9901323

[16] V. Kiselev, A. Likhoded, A. Onishchenko, Phys.Rev. D60, 014007 (1999), hep-ph/9807354

[17] A.K. Likhoded, A.I. Onishchenko (1999), hep-ph/9912425

[18] A.I. Onishchenko, Inclusive and exclusive decays of doubly heavy baryons, in 5th International Workshop on Heavy Quark Physics, Dubna, Russia, April 6-8, 2000 (2000), hep-ph/0006295

[19] V. Kiselev, A. Likhoded, Phys.Usp. 45, 455 (2002), hep-ph/0103169

[20] C.H. Chang, T. Li, X.Q. Li, Y.M. Wang, Commun.Theor.Phys. 49, 993 (2008), 0704.0016

[21] A.V. Berezhnoy, A.K. Likhoded, Phys. Atom. Nucl. 79, 260 (2016), [Yad. Fiz. 79, 151 (2016)]

[22] M. Mattson et al. (SELEX collaboration), Phys.Rev.Lett. 89, 112001 (2002), hep-ex/0208014

[23] A. Ocherashvili et al. (SELEX collaboration), Phys.Lett. B628, 18 (2005), hep-ex/0406033

[24] S.P. Ratti et al., Nucl. Phys. Proc. Suppl. 115, 33 (2003)

[25] B. Aubert et al. (BaBar collaboration), Phys.Rev. D74, 011103 (2006), hep-ex/0605075

[26] R. Chistov et al. (Belle collaboration), Phys.Rev.Lett. 97, 162001 (2006), hep-ex/0606051

[27] R. Aaij et al. (LHCb collaboration), JHEP 12, 090 (2013), 1310. 2538

[28] F.S. Yu, H.Y. Jiang, R.H. Li, C.D. Lü, W. Wang, Z.X. Zhao, Chin. Phys. C42, 051001 ( 0) (2018), 1703.09086

[29] R. Aaij et al. (LHCb collaboration), Phys. Rev. Lett. 119, 112001 (2017), 1707.01621

[30] R. Aaij et al. (LHCb collaboration), JHEP 12, 025 (2017), 1709.01769

[31] A.A. Alves Jr. et al. (LHCb collaboration), JINST 3, S08005 (2008)

[32] R. Aaij et al. (LHCb collaboration), Int. J. Mod. Phys. A30, 1530022 (2015), 1412.6352

[33] R. Aaij et al., JINST 9, P09007 (2014), 1405.7808

[34] R. Arink et al., JINST 9, P01002 (2014), 1311. 3893

[35] M. Adinolfi et al., Eur. Phys. J. C73, 2431 (2013), 1211.6759

[36] R. Aaij et al., JINST 8, P04022 (2013), 1211.3055 
[37] R. Aaij et al. (2014), LHCb-DP-2013-004/in preparation

[38] F. Archilli et al., JINST 8, P10020 (2013), 1306.0249

[39] A. Hoecker, P. Speckmayer, J. Stelzer, J. Therhaag, E. von Toerne, H. Voss, PoS ACAT, 040 (2007), physics/0703039

[40] C. Patrignani et al. (Particle Data Group), Chin. Phys. C40, 100001 (2016), and http://pdglive.lbl.gov/2017 update

[41] C.H. Chang, J.X. Wang, X.G. Wu, Comput. Phys. Commun. 177, 467 (2007), hep-ph/0702054

[42] C.H. Chang, J.X. Wang, X.G. Wu, Comput.Phys.Commun. 181, 1144 (2010), Q910. 4462

[43] T. Sjöstrand, S. Mrenna, P. Skands, Comput. Phys. Commun. 178, 852 (2008), 0710.3820

[44] T. Sjöstrand, S. Mrenna, P. Skands, JHEP 05, 026 (2006), hep-ph/0603175

[45] D.J. Lange, Nucl. Instrum. Meth. A462, 152 (2001)

[46] P. Golonka, Z. Was, Eur. Phys. J. C45, 97 (2006), hep-ph/0506026

[47] J. Allison, K. Amako, J. Apostolakis, H. Araujo, P. Dubois et al. (Geant4 collaboration), IEEE Trans.Nucl.Sci. 53, 270 (2006)

[48] S. Agostinelli et al. (Geant4 collaboration), Nucl. Instrum. Meth. A506, 250 (2003)

[49] W.D. Hulsbergen, Nucl. Instrum. Meth. A552, 566 (2005), physics/0503191

[50] G. Punzi, Sensitivity of searches for new signals and its optimization, in Statistical Problems in Particle Physics, Astrophysics, and Cosmology, edited by L. Lyons, R. Mount, R. Reitmeyer (2003), p. 79, physics/0308063

[51] T. Skwarnicki, Ph.D. thesis, Institute of Nuclear Physics, Krakow (1986), http://inspirehep.net/record/230779/DESY-F31-86-02

[52] M. Pivk, F.R. Le Diberder, Nucl.Instrum.Meth. A555, 356 (2005), physics/0402083 